\title{
CIRUGÍA DEL DESPRENDIMIENTO DE RETINA REGMATÓGENO MEDIANTE VITRECTOMÍA TRANSESCLERAL SIN SUTURA CON SISTEMA 25-G
}

\section{TRANS-SCLERAL SUTURELESS VITRECTOMY WITH A 25-G SYSTEM FOR RHEGMATOGENOUS RETINAL DETACHMENT TREATMENT}

\author{
PÉREZ-GONZÁLEZ LE ${ }^{1}$, LAJARA-BLESA JJ²
}

\section{RESUMEN}

Objetivo: Evaluar la utilidad de la vitrectomía transescleral sin sutura (VTS) con sistema 25G como alternativa en la cirugía del desprendimiento de retina regmatógeno (DRR).

Material y métodos: Se han revisado las historias clínicas de los pacientes intervenidos de DRR mediante VTS con sistema $25 \mathrm{G}$ y se han recogido los siguientes datos: edad, estado del cristalino, localización y extensión del desprendimiento, técnica quirúrgica empleada y evolución postoperatoria.

Resultados: Se han intervenido 22 ojos de 22 pacientes mediante VTS con sistema 25G. La edad media de los pacientes fue de 54,3 años. El 50\% del los ojos eran fáquicos. 8 ojos presentaban la mácula desprendida en el momento del diagnóstico. Se realizó taponamiento con SF6 en 5 y en el resto con aire. La retinopexia del desgarro se realizó con criocoagulación transescleral en 13, mediante endofotocoagulación con láser argón en 6, y se utilizaron ambos métodos en 3 . El periodo de seguimiento medio fue de 6,8 meses y las principales complicaciones que ocurrieron fueron la aparición de catara-

\section{ABSTRACT}

Purpose: The evaluation of the trans-scleral sutureless vitrectomy (TSV) efficiency using a $25 \mathrm{G}$ system as an alternative in rhegmatogenous retinal detachment (RRD) treatment.

Methods: Patients treated for RRD with TSV have been reviewed taking into account their age, phakic or pseudophakic state, RRD localization and extension, surgical technique and post-operative resolution.

Results: Twenty two eyes of 22 patients have been treated for RRD with TSV. The average age was 54.3 years. $50 \%$ of the eyes were phakic. Eight of the detachments were «macula-off» at the time of diagnosis. SF6 was used in 5 cases and air in the remainder. Retinopexia of the breaks was done with trans-scleral cryocoagulation in 13 cases, with laser endophotocoagulation in 6 cases, and with both methods in 3 cases. The average follow-up time was 6.8 months, and the main complications were cataracts in 7 of 11 phakic eyes $(63.6 \%)$ and a new retinal detachment in 4 eyes (18.2\%). The intraocular pressures were similar to those before surgery and in 18 cases $(81.8 \%)$ the visual acuity was better.

\footnotetext{
Recibido: 31/5/06. Aceptado: 12/6/07.

Servicio de Oftalmología. Hospital General Universitario Morales Meseguer. Murcia. España.

1 Licenciado en Medicina.

2 Doctor en Medicina.

Correspondencia:

Luis E. Pérez González

Hospital General Universitario Morales Meseguer

$\mathrm{C} /$. Marqués de los Vélez, s/n

30008 Murcia

España

E-mail: luiseloyperez@hotmail.com
} 
tas en 7 de los 11 ojos fáquicos $(63,6 \%)$ y un nuevo desprendimiento en 4 (18,2\%). La PIO al final del periodo de seguimiento fue similar a la previa en todos los casos y la agudeza visual final mejoró en $18(81,8 \%)$.

Conclusión: La VTS con sistema $25 \mathrm{G}$ es un método eficaz en la resolución de DRR pero que presenta limitaciones como son la necesidad de un periodo de aprendizaje y la alta incidencia de aparición de cataratas.

Palabras clave: Vitrectomía, desprendimiento de retina, complicaciones postoperatorias, cirugía, estudio retrospectivo.
Conclusion: TSV is effective in the treatment of RRD but with some limitations like the need for a learning period and a high incidence of cataracts (Arch Soc Esp Oftalmol 2007; 82: 437-442).

Key words: Vitrectomy, retinal detachment, postoperative complications, surgery, retrospective study.

\section{INTRODUCCIÓN}

El objetivo de este estudio es el de evaluar la utilidad de la vitrectomía transescleral sin sutura con sistema $25 \mathrm{G}$ como alternativa en la cirugía del desprendimiento de retina regmatógeno.

Una de las principales cuestiones que llevan al desarrollo de las técnicas quirúrgicas es el interés por conseguir el mismo resultado con el mínimo daño o lesión de los tejidos. En este sentido se han ido desarrollando en distintos campos quirúgicos técnicas que han permitido el abordaje de distintas cavidades corporales a través de pequeñas incisiones. En el caso de la ofalmología desde hace unos 15 años se han ido produciendo cada vez mayores avances en este sentido, primero para el caso de la cirugía de la catarata, que ha permitido el desarrollo de un instrumental con el que se puede realizar la extracción del cristalino opacificado a través de incisiones autosellables de $3 \mathrm{~mm}$ o menores. Más recientemente estos avances se han dirigido hacia la cirugía del segmento posterior, donde la utilización de los sistemas de vitrectomía transescleral sin sutura de 25 gauges permite la entrada en la cámara vítrea por incisiones de apenas $0,5 \mathrm{~mm}$ con un mínimo daño de los tejidos, un menor tiempo operatorio y la no necesidad de suturas para sellar las incisiones (1-5).

Fujii y colaboradores fueron los primeros en describir esta técnica así cómo presentar sus resultados $(1,2)$, definiéndola como una técnica útil y segura en muy variados casos de patología retiniana y con una serie de teóricas ventajas como son una menor manipulación y menos traumática de la conjuntiva y la esclera, menor inflamación postoperatoria intraocular, menor astigmatismo corneal (6), menores molestias postoperatorias para el paciente y una mas rápida recuperación visual (7).

En este artículo mostramos los resultados obtenidos en las intervenciones de desprendimiento de retina realizadas por un único cirujano utilizando el sistema 25-G.

\section{SUJETOS, MATERIAL Y MÉTODOS}

Revisión de las historias clínicas de los pacientes intervenidos de desprendimiento de retina mediante vitrectomía transescleral sin sutura con sistema 25 gauge (VTS). Los pacientes fueron intervenidos en tres centros distintos (uno público y dos privados) pero por un mismo cirujano. Se han recogido la edad de los pacientes, fecha del diagnóstico, situación del ojo en el momento del diagnóstico (agudeza visual, presión intraocular, estado del cristalino), tipo de desprendimiento (número de cuadrantes, número de desgarros y localización), fecha de la intervención y características (uso o no de perfluoro carbono líquido, taponamiento utilizado y criorretinopexia o endofotocoagulación de desgarros), y situación final (agudeza visual, presión intraocular, aparición o progresión de catarata tras la intervención en el caso de los pacientes fáquicos, necesidad de reintervención).

Todos los pacientes fueron intervenidos mediante VTS con el sistema 25-gauge del vitreotomo Accurus ${ }^{\circledR}$ de Alcon ${ }^{\circledR}$. En todos los casos la visualización del polo posterior se realizó mediante lente de contacto utilizando o no anillo de fijación a limbo según los casos. Todos los pacientes fueron 
anestesiados de manera locorregional mediante anestesia peribulbar. Los fondos de saco conjuntivales fueron lavados previamente a la cirugía con povidona yodada diluida al $50 \%$. Se colocaron las cánulas mediante trócares de esclerotomía en posiciones temporal inferior, para la vía de infusión, y temporal superior y nasal superior para la introducción de los instrumentos (vitreotomo, fibra óptica para iluminación, fibra óptica para láser intraocular, cánula para aspiración de perfluorocarbono líquido). La distancia de los puntos de entrada de las cánulas al limbo fue de $3,5 \mathrm{~mm}$ o $4 \mathrm{~mm}$ según fuera el paciente pseudofáquico o fáquico respectivamente. La introducción de las cánulas se realizó previa tracción de la conjuntiva hacia el limbo córneo escleral para evitar el aposicionamiento de los orificos de entrada en la conjuntiva y la esclera. En todos los casos se procedió a una vitrectomía utilizando el modo 3D, que permite el vitreotomo Accurus ${ }^{\circledR}$, con una presión de llenado de $45 \mathrm{mmHg}$, un vacío de $250 \mathrm{mmHg}$ y 1.500 cortes por segundo (cps) en el primer escalón del pedal y $500 \mathrm{mmHg}$ y $1.000 \mathrm{cps}$ al final del recorrido del pedal. Tras la vitrectomía se procedió al intercambio con aire y el aspirado del líquido subretiniano (LSR) a través del desgarro si este había sido localizado previamente. En los casos con mácula desprendida o en aquellos con bolsas de desprendimiento prominentes en los que la mácula corría riesgo de desprenderse por el empuje del aire hacia el polo posterior del líquido subretiniano, se utilizó perfluorocarbono para llevar el líquido periféricamente y posteriormente se procedió al intercambio con gas de los restos de suero y al aspirado del LSR. Una vez conseguida la aplicación de la retina se procedió al intercambio del perfluorocarbono líquido con aire y al sellado de los desgarros mediante crioretinopexia transescleral o endofotocoagulación con láser de argón, así como de las degeneraciones retinianas periféricas que se pudieran encontrar. El medio de taponamiento usado fue el aire o bien SF6 al 18\% según los casos. Tras la retirada de las cánulas se recolocó la conjuntiva en su sitio de manera que no coincidieran los orificios de entrada en ésta y la esclera y se hizo presión con una hemosteta en el punto de entrada del trocar a nivel de la esclera durante unos segundos hasta comprobar su estanqueidad. En los casos en que se apreció hipotonía tras la retirada de los trócares se inyectó vía paras plana aire o SF6 al $18 \%$ mediante una aguja de 30 gauge hasta alcanzar una presión óptima. En todos los casos se inyectó al final del proceso urbasón ${ }^{\circledR}$ subconjuntival y se instilaron gotas de tobramicina, dexametasona, y ciclopléjico y se colocó vendaje oclusivo. Se recomendó a todos los pacientes mantener reposo y posicionamiento de la cabeza según la localización del desgarro, al menos durante el período de permanencia del taponamiento dentro del ojo.

\section{RESULTADOS}

Se han recogido los datos de 22 ojos de 22 pacientes intervenidos mediante VTS con sistema 25 gauge desde abril de 2005 hasta abril de 2006. Los resultados se muestran en la tabla I. La edad media de los pacientes fue de 54,3 años. La agudeza visual en el momento del diagnóstico fue de 0,39 (rango de movimiento de manos a 1) y la PIO media de 13,3 mmHg. 11 ojos eran fáquicos y 11 pseudofáquicos. Un ojo presentaba un desprendimiento de retina total, 3 de tres cuadrantes de la retina, $10 \mathrm{de}$ dos y 8 de un cuadrante. Los desgarros se encontraban en cuadrantes superiores en 15 y en inferiores en 7. En 8 casos la mácula se encontraba desprendida en el momento del diagnóstico.

En 19 casos se utilizó PFCL para proteger la mácula o desplazar anteriormente el líquido subretiniano. En 17 casos el taponamiento se realizó con aire y en 5 con SF6. En 6 se realizó la retinopexia del desgarro mediante endofotocoagulación con láser de argón, en 13 mediante la aplicación transescleral de frío. En 3 se utilizaron ambas técnicas de manera combinada.

En ninguno de los casos fue necesario suturar las esclerotomías para mantener la presión intraocular estable al final del proceso operatorio. En el primer día del postoperatorio hubo 5 casos en los que se objetivó una presión intraocular inferior a $10 \mathrm{mmHg}$, que se recuperaron tras una semana manteniéndose estable hasta el final del periodo postoperatorio.

Uno de los ojos reintervenidos presentó un desprendimiento coroideo al día siguiente de la intervención, que se resolvió en la primera semana.

El período de seguimiento medio tras la intervención fue de 6,8 meses (1,5 a 12,5 meses), al final del cual todos los ojos presentaban la retina aplicada. De estos, en $4(18,2 \%)$ fue necesaria la reintervención (dos casos precisaron 2 reintervenciones) realizándose ésta mediante vitrectomía 20 gauges y colocación de cerclaje. El taponamiento usado en estos casos fue silicona en dos casos y SF6 al $18 \%$ en otros dos. 
Tabla I. Resultados

\begin{tabular}{|c|c|c|c|c|c|c|c|c|c|c|c|c|c|c|c|c|c|}
\hline & Ojo & Edad & $\begin{array}{l}\text { FAQ } \\
\text { PSF }\end{array}$ & $\begin{array}{l}\mathrm{AV} \\
\text { pre }\end{array}$ & $\begin{array}{l}\text { PIO } \\
\text { pre }\end{array}$ & Cuad. & Mácula & L.D. & PFCL & Tapon. & Crio. & Láser & REDR & CAT & $\begin{array}{l}\text { AV } \\
\text { post }\end{array}$ & $\begin{array}{l}\text { PIO } \\
\text { post }\end{array}$ & $\begin{array}{c}\text { PS } \\
\text { (meses) }\end{array}$ \\
\hline 1 & D & 60 & PSF & 0,1 & 15 & 3 & Off & Sup & Sí & Aire & Sí & No & No & - & 0,9 & 16 & 12,5 \\
\hline 2 & $\mathrm{D}$ & 66 & PSF & 1 & 10 & 1 & On & Sup & No & Aire & Sí & No & Sí & - & 1 & 8 & 12,5 \\
\hline 3 & I & 60 & FAQ & PL & 4 & 3 & Off & Inf & Sí & Aire & Sí & No & No & No & 0,16 & 11 & 12,5 \\
\hline 4 & I & 36 & FAQ & 0,6 & 12 & 2 & On & Sup & No & Aire & Sí & No & No & No & 0,9 & 15 & 12,0 \\
\hline 5 & I & 55 & PSF & 0,5 & 14 & 2 & On & Inf & Sí & Aire & Sí & No & Sí & - & 0,8 & 12 & 11,0 \\
\hline 6 & $\mathrm{D}$ & 54 & PSF & MM & 20 & 4 & Off & $\mathrm{T}$ & Sí & Aire & Sí & Sí & No & - & 0,4 & 20 & 9,5 \\
\hline 7 & D & 43 & FAQ & 0,7 & 12 & 2 & On & Inf & Sí & Aire & Sí & No & No & Sí & 0,6 & 12 & 9,0 \\
\hline 8 & $\mathrm{D}$ & 39 & FAQ & 0,9 & 15 & 1 & On & Sup & No & Aire & Sí & No & No & No & 1 & 15 & 8,0 \\
\hline 9 & D & 69 & FAQ & MM & 10 & 2 & Off & Sup & Sí & Aire & Sí & No & Sí & Sí & CD & 6 & 7,5 \\
\hline 10 & D & 58 & PSF & 0,2 & 17 & 3 & Off & Inf & Sí & Aire & Sí & No & No & - & 1 & 14 & 7,5 \\
\hline 11 & D & 82 & PSF & 0,2 & 17 & 2 & Off & Sup & Sí & Aire & Sí & Sí & Sí & - & MM & 10 & 7,5 \\
\hline 12 & I & 26 & FAQ & 0,5 & 10 & 1 & On & Inf & Sí & SF6 & No & Sí & No & Sí & 0,3 & 14 & 7,0 \\
\hline 13 & D & 39 & FAQ & 0,05 & 20 & 2 & Off & Sup & Sí & Aire & Sí & No & No & Sí & 0,5 & 16 & 6,0 \\
\hline 14 & I & 54 & PSF & 0,2 & 9 & 2 & On & Sup & Sí & SF6 & No & Sí & No & - & 0,9 & 13 & 6,0 \\
\hline 15 & D & 57 & FAQ & MM & 12 & 2 & Off & Sup & Sí & SF6 & Sí & Sí & No & Sí & 0,2 & 8 & 5,0 \\
\hline 16 & $\mathrm{D}$ & 45 & PSF & 0,7 & 10 & 1 & On & Inf & Sí & SF6 & No & Sí & No & - & 0,7 & 18 & 4,0 \\
\hline 17 & D & 50 & FAQ & 0,3 & 14 & 2 & On & Sup & Sí & SF6 & No & Sí & No & Sí & 0,1 & 15 & 4,0 \\
\hline 18 & D & 69 & PSF & 0,8 & 16 & 1 & On & Inf & Sí & Aire & No & Sí & No & - & 0,8 & 14 & 2,0 \\
\hline 19 & I & 68 & PSF & 0,6 & 14 & 1 & On & Sup & No & Aire & Sí & No & No & - & 0,7 & 15 & 2,0 \\
\hline 20 & I & 58 & PSF & 0,3 & 14 & 1 & On & Sup & Sí & Aire & No & Sí & No & - & 0,4 & 14 & 1,5 \\
\hline 21 & D & 46 & FAQ & 0,6 & 12 & 2 & On & Inf & Sí & Aire & Sí & No & No & No & 0,8 & 12 & 1,5 \\
\hline 22 & D & 62 & FAQ & 0,3 & 16 & 1 & On & Sup & Sí & Aire & Sí & No & No & Sí & 0,4 & 16 & 1,5 \\
\hline
\end{tabular}

AVpre $=$ Agudeza visual previa. $\mathrm{AVpost}=$ Agudeza visual al final del periodo de seguimiento. $\mathrm{CAT}=\mathrm{Aparición}$ de catarata. $\mathrm{CD}=\mathrm{contar}$ dedos. $\mathrm{Crio} .=$ crioretinopexia. $\mathrm{CUAD}=$ Número de cuadrantes afectados $\mathrm{D}=$ derecho. $\mathrm{FAQ}=$ Fáquico. $\mathrm{I}=$ izquierdo. Inf $=\mathrm{Inferior} . \mathrm{LD}=$ localización del desgarro. $\mathrm{MM}=$ movimiento de manos. PIO pre= Presión intraocular previa. PIO post=presion intraocular al final del periodo de seguimiento. $\mathrm{PFCL}=\mathrm{Perfluo}-$ rocarbono líquido. $\mathrm{PS}=$ periodo de seguimiento. $\mathrm{PSF}=$ Pseudofáquico. REDR=redesprendimiento. $\mathrm{Sup}=$ superior. TAPON= Taponamiento usado.

Los desprendimientos de retina ocurridos tras la intervención lo hicieron todos entre la segunda semana y el primer mes del periodo de seguimiento, encontrándose en todos ellos desgarros no apreciados previamente. Todos los redesprendimientos fueron ojos de pacientes intervenidos en los 6 primeros meses desde que comenzamos a utilizar esta técnica, no apareciendo ninguno en los siguientes 6 meses, ni tampoco en aquellos en los que se utilizó SF6 como taponamiento.

De los 11 pacientes fáquicos, 7 (63,6\%) desarrollaron una catarata tras la intervención. La opacidad en el cristalino varió desde la aparición de una catarata completa, en un caso, a opacidades subcapsulares posteriores en distinto grado en el resto. Estos pacientes todavía no han sido intervenidos de catarata, excepto uno que fue reintervenido de desprendimiento de retina, siendo intervenido de la catarata en el mismo tiempo operatorio.

La agudeza visual final media fue de 0,57 (rango de movimiento de manos a 1) consiguiéndose la mejoría en 16, dos quedaron igual y 4 empeoraron. La agudeza visual fue inferior a 0,1 sólo en dos casos en que fue preciso reintervenir. Los casos de pérdida de visión se dieron en 3 pacientes por la aparición de catarata y en uno por tratarse de un caso de redesprendimiento intervenido utilizando taponamiento con silicona.

\section{DISCUSIÓN}

La vitrectomía con sistema 25-gauge fue descrita hace unos 4 años por Fujii et al $(1,2)$. Éstos la describían como un sistema cuyo uso estaría indicado sobre todo en procesos que no requerían mucha manipulación intraocular, debido a sus grandes ventajas derivadas de la no necesidad de abrir la conjuntiva y de no suturar tras la intervención, como son un menor tiempo operatorio, una menor inflamación postoperatoria y un menor tiempo de recuperación visual.

En este estudio hemos descrito una serie de casos intervenidos de desprendimiento de retina mediante esta técnica. En todos estos casos se prefirió utilizar el sistema VTS de 25-G por no considerarse necesario en ninguno de ellos abrir la conjuntiva con otro fin (colocación de implantes, cerclaje escleral o 
manipulación de los músculos extraoculares). En todos los casos se trataba de ojos con desprendimiento de retina primario, de reciente aparición y sin proliferación vitreorretiniana.

En 16 casos (72\%) se utilizó como método para la pexia de los desgarros la criocoagulación. Este método se prefirió debido a que se trataba de desgarros que se encontraban en situación temporal superior, lo que dificultaba el abordaje de éstos con la sonda del láser debido a la mayor dificultad de movilización de ésta dentro del ojo por la rigidez de la cánula intaocular y por no disponer de sondas con punta dirigible hasta ese momento.

En algunas series (2-4) se han descrito casos en los que fue necesario suturar las esclerotomías para mantener la presión intraocular estable al finalizar el proceso quirúrgico, en estos casos se trataba de procesos quirúrgicos que precisaron de una gran manipulación y movilidad intraocular, y por tanto de movilización de las cánulas colocadas en las esclerotomías, lo que podría provocar un ensanchamiento de éstas. En nuestra serie no fue necesario suturar las esclerotomías en ningún caso tras la cirugía ni en los días siguientes. Los casos de hipotonía en los días siguientes a la cirugía no precisaron de ninguna intervención, al igual que en otras series (2-6), y se normalizaron en la siguiente semana siendo las presiones intraoculares al final del periodo de seguimiento similares a las preoperatorios en todos los casos. La hipotensión ocular postoperatoria se ha conseguido solucionar con la realización de esclerotomías oblicuas que permiten un autosellado más eficaz de las heridas $(8,9)$.

El caso en que se halló un desprendimiento coroideo en el día siguiente a la cirugía, pudo ser debido a hipotensión en el momento de retirar la cánulas, por no haberse detectado previamente.

Una de las principales complicaciones que aparecieron en el periodo postoperatorio fue la aparición o progresión de cataratas en 7 de los 11 ojos fáquicos intervenidos en nuestra serie $(63,6 \%)$ y en otra serie en 23 de 29 (79,3\%) (3). La localización subcapsular posterior de las opacidades y su aparición en los primeros días, hace pensar en la posibilidad de que estas fueran resultado del contacto de los instrumentos con la cápsula posterior al intentar acceder al vítreo más anterior o por el contacto de la vía de infusión con el cristalino al indentar transescleralmente.

Los resultados en cuanto a resolución del proceso fueron excelentes postoperatoriamente, consi- guiéndose la reaplicación de la retina en el postoperatorio en todos los casos. Sin embargo, entre la segunda y cuarta semana del postoperatorio en cuatro casos se produjo un nuevo desprendimiento de retina secundario a la aparición de desgarros en la parte inferior que no se objetivaron en el momento de la cirugía pese a revisar la periferia de la retina mediante indentación transescleral. Todos estos casos de redesprendimiento ocurrieron en ojos intervenidos en los 6 primeros meses desde que comenzamos a utilizar esta técnica, mientras que a partir de entonces no ha ocurrido en ningún caso hasta el momento, habiendo superado en todos las cuatro semanas de periodo de seguimiento. Esto nos lleva a pensar que estos desgarros pudieron aparecer en los siguientes días del postoperatorio, por contracciones de remanentes periféricos de vítreo, que quedaron tras una vitrectomía incompleta, por la falta de experiencia en el uso de esta técnica. Casos similares de desprendimiento de retina en grandes series de vitrectomía han sido descritos tras intervenciones de vitrectomía transescleral sin sutura con sistema de 25 -gauges $(2,3,10)$.

La agudeza visual se consiguió mejorar o mantener en 18 ojos $(81,8 \%)$. Los pacientes en que apareció catarata no han sido intervenidos todavía, por lo que los resultados visuales pueden ser mejores tras la extracción de ésta.

La vitrectomía transescleral sin sutura con sistema $25 \mathrm{G}$ es un método eficaz para intervenir los desprendimientos de retina regmatógenos de primera intención y sin proliferación vitreoretiniana, aportando grandes ventajas como son: una menor manipulación de los tejidos, un menor tiempo operatorio y un menor tiempo de recuperación visual. Como inconvenientes se debería tener en cuenta que es un sistema que presenta una mayor dificultad de acceso a vítreo periférico que otros sistemas de vitrectomía y por tanto precisa de un periodo de aprendizaje y perfeccionamiento que aumenta el riesgo de catarata por contacto de los instrumentos.

\section{BIBLIOGRAFÍA}

1. Fujii GY, De Juan E Jr, Humayun MS, Pieramici DJ, Chang TS, Awh C, et al. A new 25-gauge instrument system for transconjunctival sutureless vitrectomy surgery. Ophthalmology 2002; 109: 1807-1812.

2. Fujii GY, De Juan E Jr, Humayun MS, Chang TS, Pieramici DJ, Barnes A, et al. Initial experience using the transconjunctival sutureless vitrectomy system for vitreoretinal surgery. Ophthalmology 2002; 109: 1814-1820. 
3. Ibarra MS, Hermel M, Prenner JL, Hassan TS. Longerterm outcomes of transconjunctival sutureless 25-gauge vitrectomy. Am J Ophthalmol 2005; 139: 831-836.

4. Lakhanpal RR, Humayun MS, de Juan E Jr, Lim JI, Chong LP, Chang TS, et al. Outcomes of 140 consecutive cases of 25-gauge trnsconjunctival surgery for posterior segment disease. Ophthalmology 2005; 112: 817824.

5. Cho YJ, Lee JM, Kim SS. Vitreoretinal Surgery using transconjunctival sutureless vitrectomy. Yonsei Med J 2004; 45: 615-620.

6. Yanyali A, Celik E, Horozoglu F, Nohutcu AF. Corneal topographic changes after transconjunctival (25-gauge) sutureless vitrectomy. Am J Ophthalmol 2005; 140: 939941.
7. Kadonosono K, Yamakawa T, Uchio E, Yanagi Y, Tamaki $Y$, Araie M. Comparison of visual function after epiretinal membrana removal by 20-gauge and 25-gauge vitrectomy. Am J Ophthalmol 2006; 142: 513-515.

8. López-Guajardo L, Pareja-Esteban J, Teus-Guezala MA. Oblique sclerotomy technique for prevention of incompetent wound closure in transconjunctival 25-gauge vitrectomy. Am J Ophthalmol 2006; 141: 1154-1156.

9. Shimada H, Nakashizuka H, Mori R, Mizutani Y, Hattori T. 25-gauge scleral tunel transconjunctival vitrectomy. Am J Ophtalmol 2006; 142: 871-873.

10. Scartozzi R, Bessa AS, Gupta OP, Regillo CD. Intraoperative sclerotomy-related retinal breaks for macular surgery, 20-vs 25-gauge vitrectomy systems. Am J Ophthalmol 2007; 143: 155-156. 